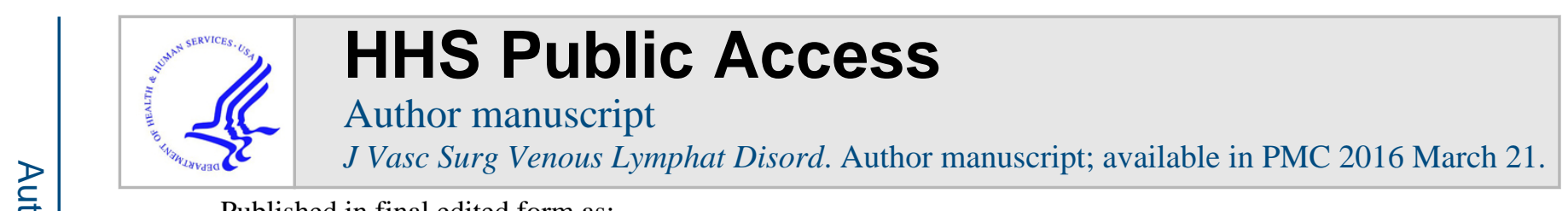

Published in final edited form as:

J Vasc Surg Venous Lymphat Disord. 2015 July ; 3(3): 290-294. doi:10.1016/j.jvsv.2014.09.010.

\title{
Concurrent venography during first rib resection and scalenectomy for venous thoracic outlet syndrome is safe and efficient
}

\author{
Alexander T. Hawkins, MD, MPH ${ }^{\mathrm{a}}$, Maria J. Schaumeier, MD ${ }^{\mathrm{a}}$, Ann D. Smith, MD, MPH ${ }^{\mathrm{a}}$, \\ Marit S. de Vos, BS ${ }^{b}$, Karen J. Ho, MD ${ }^{a}$, Marcus E. Semel, MD, MPH ${ }^{a}$, and Louis L. Nguyen, \\ MD, MBA, MPH ${ }^{\mathrm{a}, \mathrm{c}}$ \\ aDivision of Vascular \& Endovascular Surgery, Brigham \& Women's Hospital \\ ${ }^{b}$ Center for Surgery and Public Health, Brigham \& Women's Hospital \\ cHarvard Medical School
}

\begin{abstract}
Objective-Surgical treatment of acute axillosubclavian vein thrombosis from venous thoracic outlet syndrome (VTOS) traditionally involves first rib resection and scalenectomy (FRRS)

followed by interval venography and balloon angioplasty. This approach can lead to an extended need for anticoagulation and a separate anesthesia session. We present outcomes for FRRS with concurrent venography.
\end{abstract}

Methods-Retrospective chart review was performed for consecutive patients undergoing FRRS with concurrent venography for VTOS from February 2007 to April 2014. Venography was performed immediately after FRRS with the arm in neutral and provocative positions. The primary outcomes of this study were primary and primary-assisted patency. Secondary outcomes included whether concurrent venography resulted in modification of the procedure, postoperative anticoagulation use, and postoperative complications.

Results-Thirty patients underwent first rib resection with venography with a mean follow-up time of 24.4 months. The mean age was 29.5 years (range, 17-52 years), and 17 (56.7\%) were

\footnotetext{
Reprint requests: Louis L. Nguyen, MD, MBA, MPH, Division of Vascular and Endovascular Surgery, Brigham \& Women's Hospital, 75 Francis St, Boston, MA 02115 (1lnguyen@partners.org).

Author conflict of interest: none.

Presented at the Fortieth Annual Meeting of the New England Society for Vascular Surgery, Stowe, Vt, September 27-29, 2013. The editors and reviewers of this article have no relevant financial relationships to disclose per the Journal policy that requires reviewers to decline review of any manuscript for which they may have a conflict of interest.

AUTHOR CONTRIBUTIONS

Conception and design: AH, MJS, MD, KH, MES, LN

Analysis and interpretation: $\mathrm{AH}$

Data collection: $\mathrm{AH}$

Writing the article: AH, MJS, AS, MD, KH, MES, LN

Critical revision of the article: AH, MJS, AS, MD, KH, MES, LN

Final approval of the article: AH, MJS, AS, MD, KH, MES, LN

Statistical analysis: $\mathrm{AH}, \mathrm{LN}$

Obtained funding: LN

Overall responsibility: $\mathrm{LN}$
} 
female. All were maintained on anticoagulation before the procedure. Concurrent venography resulted in modification of the procedure in 28 patients (93.3\%). Of these, 27 patients (96.4\%) underwent balloon angioplasty and two patients $(7.1 \%)$ underwent further rib resection. Twenty patients (66.7\%) were discharged after the procedure with no anticoagulation. For those receiving postoperative anticoagulation for persistent minor thrombus, median time for anticoagulation duration was 5.0 months (range, 0.8 and 16.7 months). Two patients $(6.7 \%)$ had postoperative bleeding requiring thoracentesis or video-assisted thoracoscopic evacuation of hemothorax. One patient (3.3\%) suffered rethrombosis and was successfully lysed open, resulting in a 2-year subclavian vein (SCV) primary patency of $96.7 \%$ and primary-assisted patency of $100 \%$. No patients required reoperation for VTOS, and all reported improvements in symptoms. Three patients (10.0\%) later underwent prophylactic first rib resection on the contralateral side for symptoms and SCV stenosis.

Conclusions-FRRS with concurrent venography is a safe procedure for VTOS that allows effective intraoperative modification of the surgical plan, resulting in excellent patency of the SCV, early cessation of anticoagulation, and durable relief of symptoms.

Paget-Schroetter syndrome, otherwise known as effort-induced thrombosis, is an acute thrombosis of the subclavian or axillary vein. This distinct and unique subset of thoracic outlet syndrome was reported by Sir Paget in 1875 and von Schroetter in 1884 and named by Hughes in $1949 .{ }^{1}$ Patients tend to be young, and many are active and athletic. These patient characteristics, along with findings at surgery, suggest that the scarring that leads to the thoracic outlet syndrome is a result of repetitive movement. Venous thoracic outlet syndrome (VTOS) is the second most common of the thoracic outlet syndromes, representing $5 \%$ of all cases of thoracic outlet syndrome. ${ }^{2,3}$

Consensus on standard treatment of acute VTOS remains elusive. A general algorithm of catheter-directed thrombolysis, surgical first rib resection and scalenectomy (FRRS), and follow-up venography has been accepted, but many subtleties remain undefined. ${ }^{4}$ One of these is the timing of follow-up venography. Multiple proposed pathways recommend a delay of 2 to 3 weeks between first rib resection and venography. ${ }^{4-11}$ Others have described performing concurrent venography immediately after the FRRS. ${ }^{12}$ The aim of this study was to demonstrate the safety and effectiveness of concurrent venography during FRRS for VTOS.

\section{METHODS}

\section{Case identification}

All consecutive patients treated with FRRS for acute VTOS from February 2007 to April 2014 at the Brigham and Women's Hospital by a single surgeon were retrospectively reviewed. Patients with neurogenic or arterial thoracic outlet syndrome were excluded, as were patients with catheter- or wire-induced upper extremity axillosubclavian vein thrombosis or chronic (>4 weeks without anticoagulation) thrombosis. Patient demographics and perioperative and postoperative data were collected. The primary outcomes of this study were primary and primary-assisted patency. Secondary outcomes included whether concurrent venography resulted in modification of the procedure, postoperative 
anticoagulation use, and postoperative complications. Modification of the procedure was defined as any additional venolysis, venoplasty, or further rib resection performed after venography. This study was reviewed and approved by the Partners Institutional Review Board with specific waiver of the need for individual patient consent because of the retrospective nature of the study.

\section{Treatment protocol}

Patients with acute subclavian vein (SCV) thrombosis were treated with a standard protocol of initial venography with catheter-guided and mechanical thrombolysis. The diagnosis of VTOS was confirmed by a venogram with arm abduction that showed either cessation of SCV flow or thrombosis of the vein. All patients treated at our institution underwent thrombolysis with catheter-based tissue plasminogen activator and pulse AngioJet. After the initial venogram, patients were anticoagulated with low-molecular-weight heparin while awaiting surgery. Patients then underwent surgical decompression, usually 2 weeks after thrombolysis. Surgical decompression was performed under general endotracheal anesthesia and consisted of middle and anterior scalenectomy along with FRRS. The approach was paraclavicular and used combined supraclavicular and infraclavicular incisions. After rib resection, extensive, circumferential external venolysis was performed on the SCV. All patients then underwent venography with digital subtraction fluoroscopy during the same procedure. Venography was performed through the basilic or cephalic vein, which was accessed with a micropuncture needle under ultrasound guidance. Venography was performed with the arm in rest adduction (Fig, $A$ ) as well as in abduction position (Fig, $B$ ) to evaluate for residual stenosis and external compression. If stenosis was identified, balloon angioplasty was performed with the administration of systemic heparin (Fig, 1C).

Alternatively, if the venogram identified any external compression, further rib resection or external venolysis was performed. At the end of the intervention, completion venography was performed to ensure that the stenosis had been relieved. Closed suction Blake drains were left in all patients. Postoperatively, patients were started or maintained on aspirin for 1 year. Anticoagulation was continued only in cases in which there was residual thrombus in the SCV or other venous segments not treated with FRRS (brachial or axillary veins). Postoperatively, patients were instructed not to lift the ipsilateral arm above the head for 2 weeks and not to lift more than 10 pounds for 4 weeks. Activity was slowly liberalized thereafter. Patients underwent follow-up at 1 month, 6 months, and 12 months and then on a yearly basis, consisting of bilateral VTOS ultrasound examinations with the arms in both adduction and abduction. Follow-up venography was performed only if the finding on ultrasound was abnormal.

\section{Statistical analysis}

Continuous and categorical variables are expressed as mean \pm range and proportions, respectively, throughout the manuscript with the exception of continuous variables with grossly skewed distributions, which are reported as the median with the interquartile range. SAS statistical software (version 9.2; SAS Institutes Inc, Cary, NC) was used for all analysis. 


\section{RESULTS}

Thirty patients underwent FRRS during the study period (2007-2014), and all received concurrent venography. The mean age was 29.5 years (range, 17-52 years), and 17 (56.7\%) were female (Table I). No patients were discovered to have an underlying hypercoagulable state. Sixteen patients (53.3\%) underwent thrombolysis with catheter-based tissue plasminogen activator and pulse AngioJet at our institution. The balance underwent thrombolysis at an outside institution. All were maintained on anticoagulation before the procedure. The median wait time between thrombolysis and FRRS was 3.2 weeks (range, $0.7-20$ weeks), with the outlier being a patient who was maintained on anticoagulation before presentation to our center. Concurrent intraoperative venography resulted in modification of the procedure in 28 patients (93.3\%). Of these, 27 patients $(96.4 \%)$ underwent balloon angioplasty for persistent thrombosis, and two patients (6.7\%) underwent resection of a residual rib segment for persistent stenosis.

There were no deaths (Table II). Twenty patients (66.7\%) were discharged from the hospital after the procedure with no anticoagulation. For those receiving postoperative anticoagulation for persistent minor thrombus, median time for anticoagulation duration was 5.0 months (range, 0.8 and 16.7 months). Two early patients (6.7\%) in the study cohort had postoperative bleeding requiring thoracentesis or video-assisted thoracoscopic evacuation of hemothorax. There were no other complications, such as wound infection, lymph leak, brachial plexus injury, winged scapula (from injury to the long thoracic nerve), or paralyzed hemidiaphragm (from injury to the phrenic nerve).

The overall mean follow-up time was 24.2 months. One patient (3.3\%) suffered rethrombosis on postoperative day 20 and was successfully lysed open. The patient was not anticoagulated. The 2-year SCV primary patency rate was $96.7 \%$, and the 2-year primaryassisted patency rate was $100 \%$. No patients required ipsilateral reoperation for VTOS. All reported improvements in symptoms (100\%) and were back to their baseline status at follow-up. Many of the patients were competitive athletes, and all resumed their sport. Three patients $(10.0 \%)$ underwent prophylactic FRRS on the contralateral side for symptoms and $\mathrm{SCV}$ stenosis at a subsequent surgery.

\section{DISCUSSION}

This study describes 30 patients who successfully underwent FRRS and concurrent venography for VTOS. In this series, the 2-year SCV primary patency rate was $96.7 \%$, and the 2-year primary-assisted patency rate was $100 \%$. Concurrent venography resulted in modification of the procedure in $93.3 \%$ of the cases; $66.7 \%$ of patients were able to be discharged without anticoagulation.

The treatment algorithm of early catheter-directed thrombolytic therapy followed by FRRS was first proposed by Kunkel and Machleder in $1989 .{ }^{13}$ Their original protocol described a 3 -month waiting period before first rib resection that led to a $10 \%$ rethrombosis rate. ${ }^{14}$

Multiple authors have since shown the efficacy of and improved outcomes with immediate 
thoracic outlet decompression after thrombolysis. ${ }^{8,15-19}$ The issue of post-FRRS venography, however, remains under debate.

Molina et al ${ }^{10}$ advocate performing a vein patch, which they argue avoids the need for venography and venoplasty. Their results of $100 \%$ patency are difficult to argue with, but this study demonstrates that similar outcomes can be achieved without a vein patch, which likely increases operative time and complexity. Other authors, including Sanders and Hammond, ${ }^{20}$ Divi et al, ${ }^{21}$ and Doyle et al, ${ }^{16}$ do not routinely perform post-FRRS venography. Still other authors promote an algorithm that includes venography 2 to 3 weeks after FRRS. ${ }^{5-8}$ Stenosis rates at follow-up venography range from $55 \%$ to $64 \% .6,7$

Schneider et $\mathrm{al}^{12}$ first proposed concurrent venography at the time of FRRS. They described $100 \%$ technical success and 1-year primary and secondary vein patency rates of $92 \%$ and $96 \%$, respectively. Melby et $\mathrm{al}^{22}$ have also described success with this approach. This study adds to the literature in our description of how concurrent venography changes the operative plan. Early in the protocol, all patients received anticoagulation after FRRS. Because of the occurrence of bleeding complications, we modified the protocol to not systemically anticoagulate patients who had no significant thrombus or stenosis seen on the intraoperative venogram. The reduction in postoperative anticoagulation use was a major factor in reducing bleeding complications in our later patients.

This study's results highlight the potential for reducing both the number of readmissions for separate procedures and the need to routinely anticoagulate patients postoperatively. The intraoperative venogram also provided an immediate assessment of the treatment results and allowed additional maneuvers if necessary (resection of additional rib segments, additional venolysis, or vein patching). Intra-operative fluoroscopy allows the surgeon to confirm the extent of the rib resection because anatomic variability and difficult precise visualization through the small incisions can lead to incomplete rib resection. Intraoperative venography also permits immediate access to the SCV if balloon angioplasty results in a tear of the vein, an option not possible in venography performed days or weeks after first rib resection. We believe the treatment options afforded by intraoperative venography contribute to the safety and efficacy of FRRS.

This study has a number of important limitations that warrant mention. First, this is a singlecenter, single-surgeon retrospective study with a modest patient cohort. However, the majority of series on VTOS are small, and this speaks to the need for larger, multiinstitutional studies. Second, the primary and secondary SCV patency rates reported are based on ultrasound evaluation of the SCV rather than on venography. Because ultrasound is recognized to be less accurate than venography at assessing patency of the SCV, it is unclear if this is an optimal method for evaluating results. However, we believe that routine venography in asymptomatic patients with ultrasound studies showing patent veins is not justified. We hope that future work will also demonstrate the efficacy of ultrasound as a screening tool for follow-up of patients with thoracic outlet syndrome. Last, the lack of an appropriate control group denies any ability to comment on the efficacy of this approach compared with the current alternative practice of follow-up venography at 2 to 3 weeks or 
no venography at all. This again highlights the need for further study in this area, preferably in a prospective or randomized controlled trial setting.

These limitations notwithstanding, this series presents a more efficient method to treat VOTS that theoretically results in fewer overall procedures and reduces the need for continued anticoagulation. The outcomes we describe are excellent and in skilled hands should be easily reproducible.

\section{CONCLUSIONS}

FRRS with concurrent venography is a safe procedure for VTOS that allows effective intraoperative modification of the surgical plan, resulting in excellent SCV patency, early cessation of anticoagulation, and durable symptom relief.

\section{Acknowledgments}

A.T.H and A.D.S. are supported by a grant from the Brigham \& Women's Center for Surgery and Public Health Arthur Tracy Cabot Fellowship. A.T.H is also supported by the NIH NHLBI T32 (HL007734) Harvard/Longwood Vascular Surgery Training Program. A.D.S. is also supported by the Leah Carpenter du Pont Vascular Surgery Fellowship. M.J.S. is supported by a grant from the Freiwillige Akademische Gesellschaft, Basel, Switzerland.

\section{References}

1. Hughes ES. Venous obstruction in the upper extremity. Br J Surg. 1948; 36:155-63. [PubMed: 18895122]

2. McCleery RS, Kesterson JE, Kirtley JA, Love RB. Subclavius and anterior scalene muscle compression as a cause of intermittent obstruction of the subclavian vein. Ann Surg. 1951; 133:588-602. [PubMed: 14830129]

3. Sanders RJ, Hammond SL, Rao NM. Diagnosis of thoracic outlet syndrome. J Vasc Surg. 2007; 46:601-4. [PubMed: 17826254]

4. Brooke BS, Freischlag JA. Contemporary management of thoracic outlet syndrome. Curr Opin Cardiol. 2010; 25:535-40. [PubMed: 20838336]

5. Thompson JF, Winterborn RJ, Bays S, White H, Kinsella DC, Watkinson AF. Venous thoracic outlet compression and the Paget-Schroetter syndrome: a review and recommendations for management. Cardiovasc Intervent Radiol. 2011; 34:903-10. [PubMed: 21448772]

6. Guzzo JL, Chang K, Demos J, Black JH, Freischlag JA. Preoperative thrombolysis and venoplasty affords no benefit in patency following first rib resection and scalenectomy for subacute and chronic subclavian vein thrombosis. J Vasc Surg. 2010; 52:658-62. discussion: 662-3. [PubMed: 20816319]

7. de Leon RA, Chang DC, Hassoun HT, Black JH, Roseborough GS, Perler BA, et al. Multiple treatment algorithms for successful outcomes in venous thoracic outlet syndrome. Surgery. 2009; 145:500-7. [PubMed: 19375608]

8. Caparrelli DJ, Freischlag J. A unified approach to axillosubclavian venous thrombosis in a single hospital admission. Semin Vasc Surg. 2005; 18:153-7. [PubMed: 16168891]

9. Molina JE, Hunter DW, Dietz CA. Protocols for Paget-Schroetter syndrome and late treatment of chronic subclavian vein obstruction. Ann Thorac Surg. 2009; 87:416-22. [PubMed: 19161749]

10. Molina JE, Hunter DW, Dietz CA. Paget-Schroetter syndrome treated with thrombolytics and immediate surgery. J Vasc Surg. 2007; 45:328-34. [PubMed: 17264012]

11. Molina JE. Regarding "combination treatment of venous thoracic outlet syndrome: open surgical decompression and intraoperative angioplasty. J Vasc Surg. 2005; 42:593. author reply: 593. [PubMed: 16171617] 
12. Schneider DB, Dimuzio PJ, Martin ND, Gordon RL, Wilson MW, Laberge JM, et al. Combination treatment of venous thoracic outlet syndrome: open surgical decompression and intraoperative angioplasty. J Vasc Surg. 2004; 40:599-603. [PubMed: 15472583]

13. Kunkel JM, Machleder HI. Treatment of Paget-Schroetter syndrome. A staged, multidisciplinary approach. Arch Surg. 1989; 124:1153-7. discussion: 1157-8. [PubMed: 2529837]

14. Machleder HI. Evaluation of a new treatment strategy for Paget-Schroetter syndrome: spontaneous thrombosis of the axillary-subclavian vein. J Vasc Surg. 1993; 17:305-15. discussion: 316-7. [PubMed: 8433426]

15. Urschel HC Jr, Razzuk MA. Paget-Schroetter syndrome: what is the best management? Ann Thorac Surg. 2000; 69:1663-8. discussion: 1668-9. [PubMed: 10892903]

16. Doyle A, Wolford HY, Davies MG, Adams JT, Singh MJ, Saad WE, et al. Management of effort thrombosis of the subclavian vein: today's treatment. Ann Vasc Surg. 2007; 21:723-9. [PubMed: 17923385]

17. Lee MC, Grassi CJ, Belkin M, Mannick JA, Whittemore AD, Donaldson MC. Early operative intervention after thrombolytic therapy for primary subclavian vein thrombosis: an effective treatment approach. J Vasc Surg. 1998; 27:1101-7. discussion: 1107-8. [PubMed: 9652472]

18. Azakie A, McElhinney DB, Thompson RW, Raven RB, Messina LM, Stoney RJ. Surgical management of subclavian-vein effort thrombosis as a result of thoracic outlet compression. J Vasc Surg. 1998; 28:777-86. [PubMed: 9808844]

19. Angle N, Gelabert HA, Farooq MM, Ahn SS, Caswell DR, Freischlag JA, et al. Safety and efficacy of early surgical decompression of the thoracic outlet for Paget-Schroetter syndrome. Ann Vasc Surg. 2001; 15:37-42. [PubMed: 11221942]

20. Sanders RJ, Hammond SL. Subclavian vein obstruction without thrombosis. J Vasc Surg. 2005; 41:285-90. [PubMed: 15768011]

21. Divi V, Proctor MC, Axelrod DA, Greenfield LJ. Thoracic outlet decompression for subclavian vein thrombosis: experience in 71 patients. Arch Surg. 2005; 140:54-7. [PubMed: 15655206]

22. Melby SJ, Vedantham S, Narra VR, Paletta GA Jr, Khoo-Summers L, Driskill M, et al. Comprehensive surgical management of the competitive athlete with effort thrombosis of the subclavian vein (Paget-Schroetter syndrome). J Vasc Surg. 2008; 47:809-20. discussion: 821. [PubMed: 18280096] 

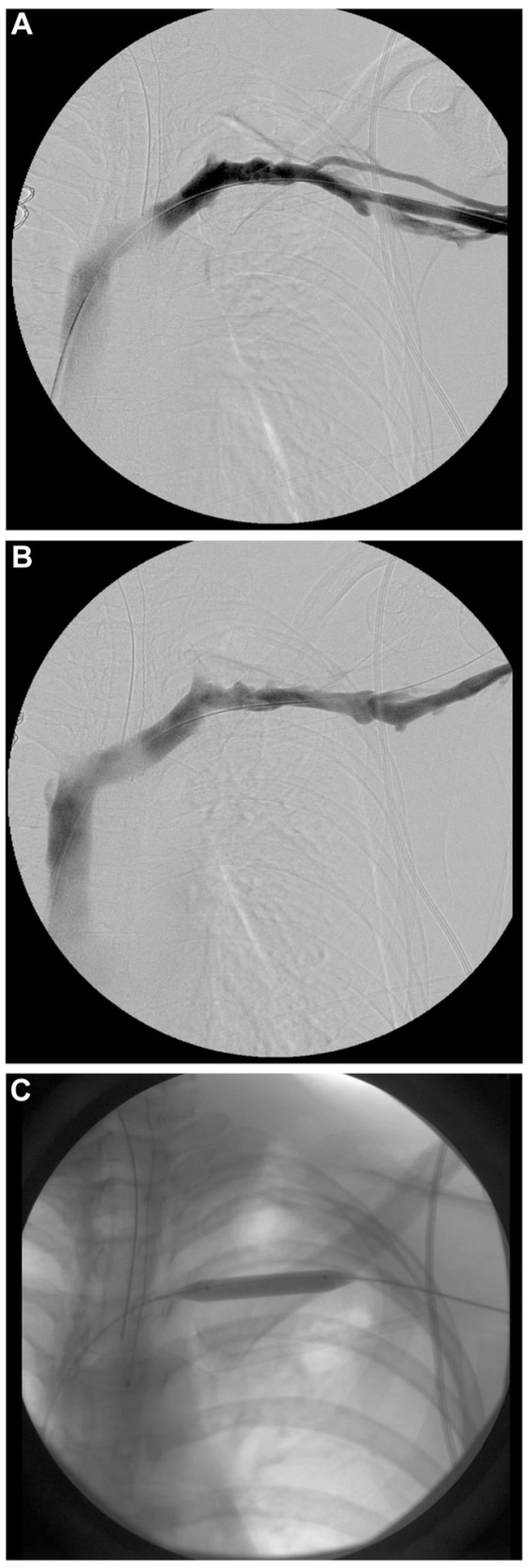

Fig.

A, Subclavian vein (SCV) in adduction after rib resection. B, SCV in abduction after rib resection. C, Balloon angioplasty. 


\section{Table I}

Patient demographics

\begin{tabular}{lc}
\hline Variables & No. $(\%)(\boldsymbol{N}=\mathbf{3 0})$ \\
\hline Female & $17(56.7)$ \\
Mean age, years (range) & $29.5(17-52)$ \\
Right-hand dominant & $29(96.7)$ \\
Right upper extremity & $24(80.0)$ \\
Catheter-directed venolysis & $30(100)$ \\
Preoperative anticoagulation & $30(100)$ \\
\hline
\end{tabular}

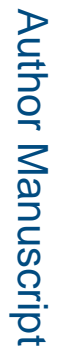

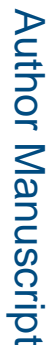

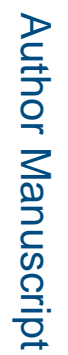

J Vasc Surg Venous Lymphat Disord. Author manuscript; available in PMC 2016 March 21. 


\section{Table II}

\section{Outcomes}

\begin{tabular}{lc}
\hline Outcomes & No. $(\boldsymbol{\%})(\boldsymbol{N}=\mathbf{3 0})$ \\
\hline Operative mortality & $0(0.0)$ \\
Discharged without anticoagulation & $20(66.7)$ \\
Median duration of anticoagulation, months (range) & $5.0(0.8-16.7)$ \\
Bleeding complication & $2(6.7)$ \\
Rethrombosis & $1(3.3)$ \\
Two-year primary patency & $29(96.7)$ \\
Two-year primary-assisted patency & $30(100)$ \\
\hline
\end{tabular}

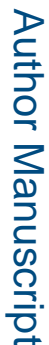

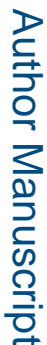

